\title{
BMJ open Uses and misuses of the STROBE statement: bibliographic study
}

\author{
Bruno R da Costa, ${ }^{1}$ Myriam Cevallos, ${ }^{1,2}$ Douglas G Altman, ${ }^{3}$ Anne W S Rutjes, ${ }^{1}$ \\ Matthias Egger ${ }^{1}$
}

To cite: da Costa BR, Cevallos M, Altman DG, et al. Uses and misuses of the STROBE statement: bibliographic study. BMJ Open 2011;1:e000048. doi:10.1136/bmjopen-2010000048

- Prepublication history for this paper is available online. To view these files please visit the journal online (http:// bmjopen.bmj.com).

Received 22 December 2010 Accepted 4 February 2011

This final article is available for use under the terms of the Creative Commons Attribution Non-Commercial 2.0 Licence; see http://bmjopen.bmj.com

${ }^{1}$ Institute of Social \& Preventive Medicine, University of Bern, Bern, Switzerland

${ }^{2}$ Clinical Trials Unit Bern, Bern University Hospital, Bern, Switzerland ${ }^{3}$ Centre for Statistics in Medicine, University of Oxford, Oxford, UK

Correspondence to Dr Bruno R da Costa; bdacosta@ispm.unibe.ch

\section{ABSTRACT}

Objectives: Appropriate reporting is central to the application of findings from research to clinical practice. The Strengthening the Reporting of Observational Studies in Epidemiology (STROBE) recommendations consist of a checklist of 22 items that provide guidance on the reporting of cohort, case-control and cross-sectional studies, in order to facilitate critical appraisal and interpretation of results. STROBE was published in October 2007 in several journals including The Lancet, BMJ, Annals of Internal Medicine and PLOS Medicine. Within the framework of the revision of the STROBE recommendations, the authors examined the context and circumstances in which the STROBE statement was used in the past. Design: The authors searched the Web of Science database in August 2010 for articles which cited STROBE and examined a random sample of 100 articles using a standardised, piloted data extraction form. The use of STROBE in observational studies and systematic reviews (including meta-analyses) was classified as appropriate or inappropriate. The use of STROBE to guide the reporting of observational studies was considered appropriate. Inappropriate uses included the use of STROBE as a tool to assess the methodological quality of studies or as a guideline on how to design and conduct studies.

Results: The authors identified 640 articles that cited STROBE. In the random sample of 100 articles, about half were observational studies $(32 \%)$ or systematic reviews $(19 \%)$. Comments, editorials and letters accounted for $15 \%$, methodological articles for $8 \%$, and recommendations and narrative reviews for $26 \%$ of articles. Of the 32 observational studies, $26(81 \%)$ made appropriate use of STROBE, and three uses (10\%) were considered inappropriate. Among 19 systematic reviews, $10(53 \%)$ used STROBE inappropriately as a tool to assess study quality.

Conclusions: The STROBE reporting recommendations are frequently used inappropriately in systematic reviews and meta-analyses as an instrument to assess the methodological quality of observational studies.

\section{INTRODUCTION}

Appropriate reporting concerns the clear and complete presentation of what was planned, carried out and found in a particular study. Inappropriate reporting makes

\section{ARTICLE SUMMARY}

\section{Article focus}

- Appropriate reporting is central for the proper application of findings from clinical research into clinical practice.

- The Strengthening the Reporting of Observational Studies in Epidemiology (STROBE) recommendations aim to provide guidance to authors on how to improve the reporting of observational studies to facilitate critical appraisal and interpretation of results.

- We examined the reasons for citing STROBE and found that most observational studies used STROBE as a reporting guideline, while about half of systematic reviews used STROBE as a tool to assess the methodological quality of the studies.

\section{Key messages}

- Our study provides further evidence that authors of systematic reviews inappropriately use reporting guidelines to assess methodological study quality. Given the identified common misuse of STROBE, we discuss possible reasons and potential pitfalls of such misuse.

Strengths and limitations of this study

- We conducted a systematic review of the literature to address a relevant and insufficiently discussed issue concerning misuses of reporting guidelines. One of the main concerns of such misuse is the potential introduction of bias into systematic reviews and meta-analysis.

- A limitation of our findings is the fact that we included only articles which cited STROBE. This may have resulted in a selection bias, since some researchers may use STROBE in their study and mention it in their manuscript but do not formally cite it.

the sound application of study findings into clinical practice difficult, if not impossible. For instance, clinicians are often faced with reports of studies which do not provide enough detail of interventions for them to be implemented in clinical practice. ${ }^{1}$ Inadequate reporting may also be a problem for the synthesis of evidence. It is common for systematic reviewers or meta-analysts to 
exclude otherwise eligible studies owing to incomplete reporting. Given the human and financial resources needed to conduct clinical studies, it is clear that inadequate reporting has also important ethical and moral implications. ${ }^{2}$

To help improve the reporting of key items, reporting guidelines have been developed for various types of research design. The positive effect of reporting guidelines on the quality of reporting has been documented in several reviews. ${ }^{3-7}$ The Strengthening the Reporting of Observational Studies in Epidemiology (STROBE) Statement was developed in a collaborative effort of epidemiologists, methodologists, statisticians, researchers and journal editors to improve the reporting of observational studies, including cohort, case-control and cross-sectional studies. The initiative was established in 2004 and subsequently developed in several meetings. STROBE was published in October 2007 simultaneously in several journals including leading biomedical journals such as The Lancet, BMJ, Annals of Internal Medicine and PLoS Medicine. The STROBE recommendations are accompanied by an explanation and elaboration document (E\&E) which explains in detail the rationale of each item and provides examples of transparent reporting from published articles. To date, the STROBE Statement is endorsed by over 100 journals as well as by the International Committee of Medical Journal Editors. ${ }^{8}$

The purpose of the STROBE Statement is to ensure clear presentation of what was planned, done, and found in an observational study' and the authors of STROBE clearly stress that 'the recommendations are not prescriptions for setting up or conducting studies, nor do they dictate methodology or mandate a uniform presentation. ${ }^{9}$ Notwithstanding the clear statement of the purpose of STROBE by its authors, some journal editors are concerned that the STROBE recommendations may be inappropriately used as an assessment tool to judge study quality or that researchers may use STROBE as a guideline to set up or conduct observational studies. ${ }^{10}$

In the present study, we examined in which circumstances and context STROBE has been cited by the researchers (how, where and why), and the background of researchers who cited STROBE. We considered the use of STROBE other than in relation to its original purpose as a reporting guideline for observational studies. Our results were presented and discussed during the August 2010 STROBE group meeting, when the group met to discuss a possible revision of the STROBE recommendations in light of the group's experience, anecdotal reports, feedback from STROBE users and new evidence.

\section{METHODS}

\section{Literature search}

On 12 August 2010, we conducted a two-step literature search in the Web of Knowledge database (http://www. isiknowledge.com/). The first step consisted of a search for STROBE publications. Our search strategy to identify
STROBE publications contained words used in the title of the STROBE article and author name (appendix 1). This first search was conducted in order to set up the second search, where we identified articles which had cited any of the STROBE publications identified in the first step, using the 'create citation report' tool available in the Web of Knowledge database.

\section{Article selection}

Eligibility criteria for our first search (ie, search for STROBE publications) consisted of any STROBE publication regardless of language or version of publication (ie, checklist or E\&E). We excluded the Strengthening the Reporting of Genetic Association Studies (STREGA) recommendations, which is an extension of STROBE to genetic epidemiology.

There were no eligibility restrictions for our second search (ie, search of articles which cited STROBE). Any publication type, such as comments, editorials, systematic reviews or observational research, was eligible for inclusion. We then randomly selected a sample of 100 articles from the final list of articles which cited STROBE for detailed assessment.

\section{Data collection}

We collected the following information for STROBE publications identified through our initial search: journal in which STROBE was published, language of publication and number of citations which STROBE received per year.

For the data extraction from articles which cited STROBE, a data-extraction form was developed by the authors and piloted by two reviewers (BRdC and MC) on a sample of 40 studies which were not part of the randomly selected articles. A single reviewer (BRdC) then conducted data extraction on a randomly selected sample of 100 articles for the following items: verbatim text of sentence(s) which included STROBE citation; reason for citation; STROBE article cited; type of article that cited STROBE (observational research; systematic review or meta-analysis; comment, editorial or letter to the editor; methodological article; or recommendations or narrative review); and background of researchers. Categories for 'reason of citation' were defined during the piloting of the data-extraction form, and additional categories were defined during data extraction as necessary. Whenever the data extractor (BRdC) was in doubt about the classification of an article, he discussed it with other authors until a decision was reached. In case of disagreements, the final decision was made by the senior epidemiologist (ME).

\section{Classification of reasons for citation}

Results of our search for articles which cited STROBE are presented narratively. Our sample of 100 randomly selected articles were classified into eight groups according to reason for citation: STROBE used as a reporting guideline; STROBE used as a methodological (ie, research design or conduct) guideline; STROBE used 
as an assessment tool of reporting quality; STROBE used as an assessment tool of methodological quality; STROBE cited to stress the importance of reporting guidelines; 'unclear'; and 'other.'

We considered the use of STROBE in reports of observational research to be appropriate when STROBE was used as a reporting guideline to ensure a clear and complete report of the study's design, conduct and findings. In this case, it was clear from the text that one or more items of STROBE were used to guide the reporting of the study. Our definition of appropriate use of STROBE is in line with the stated intentions of its authors 'solely to provide guidance on how to report research well' and 'not prescriptions for designing or conducting studies. ${ }^{9}$ Accordingly, we considered inappropriate the use of STROBE as a methodological guideline (ie, guideline to design or conduct observational research or as an assessment tool of methodological quality of publications reporting observational research). An example of what we considered inappropriate is the use of STROBE to assign an overall score of the methodological quality of a study. As stressed by the authors, STROBE was not developed to explain how research should be done, and so cannot validly be used to assess methodological quality. In box 1, we present text examples of appropriate and inappropriate uses of STROBE. The remainder of the citations which did not fall into either category were considered neutral, that is, neither appropriate nor inappropriate. Only observational research, systematic reviews or meta-analyses could be classified as appropriate or inappropriate.

For exploratory purposes, we classified articles which appropriately and inappropriately used STROBE according to the affiliation of the authors as a proxy for their background. Authors affiliated with epidemiology or public-health departments were assumed to have methodological training in epidemiological research. Owing to the identified large proportion of systematic reviews which inappropriately cited STROBE, we examined these reviews further, extracting information on the language of publication (English or other) and type of journal (specialist or general medical journal).

\section{RESULTS}

\section{Search for STROBE publications}

We identified 16 STROBE publications. Eleven of these publications were published in English, three in Spanish and two in German. Ten of these publications were published in 2007, five in 2008 and one in 2009. Table 1 displays the journals where STROBE was published, the number of citations received, the impact factor of the journal, the language of the publication and the date of the publication.

\section{Search for articles which cited STROBE}

We identified 643 citations of STROBE since its first publication in October 2007 until August 2010.
Box 1 Text examples of appropriate and inappropriate use of Strengthening the Reporting of Observational Studies in Epidemiology (STROBE)

Based on the original purpose of STROBE, we consider the following text examples as appropriate or inappropriate use of STROBE

\section{Appropriate}

- 'The reporting of this study conforms to the STROBE statement. ${ }^{11}$

n 'The STROBE guidelines were used to ensure the reporting of this observational study. ${ }^{12}$

n 'The paper is reported following the STROBE statement. ${ }^{13}$

\section{Inappropriate}

- 'This prospective study was designed following recommendation of the STROBE statement. ${ }^{14}$

- 'The analysis followed the principles of the Strengthening the Reporting of Observational Studies in Epidemiology initiative. ${ }^{, 15}$

- 'The quality of selected studies was assessed using a modified version of the STROBE statement. ...The STROBE was modified by adding questions about the serologic method used to confirm dengue diagnosis, use of viral isolation, and whether the study was based on a single dengue outbreak or transmission season. Use of viral isolation increased the score whereas single outbreak studies received no additional points. The quality score was the number of items from the STROBE checklist addressed as a percentage of the total number of items applicable (minimum of 23 and maximum of 25). Studies with a quality assessment below $50 \%$ were excluded. ${ }^{16}$

Two-hundred and four citations $(32 \%)$ concerned the STROBE E\&E document, and 439 (68\%) citations concerned the STROBE checklist. The STROBE checklist published in The Lancet was the most cited, receiving $110(17 \%)$ citations. The number of citations was associated with the impact factor of the journal as shown in table 1, the main exception being the Journal of Clinical Epidemiology, which received a large number of citations despite a lower journal impact factor.

Half of the articles in our sample of 100 randomly selected articles were observational studies $(32 \%)$ or systematic reviews/meta-analyses (19\%). Table 2 displays the characteristics of these 51 research articles identified in our random sample.

Comments, editorials and letters accounted for $15 \%$ of the articles, methodological articles for $8 \%$, and recommendations and narrative reviews for $26 \%$. Of the 32 observational studies assessed, $26(81 \%)$ made an appropriate use of STROBE, and three (10\%) uses were considered inappropriate. Of the 19 systematic reviews or meta-analyses assessed, $10(53 \%)$ made an inappropriate use of STROBE. Thus, of the 51 research articles, $26(51 \%)$ used STROBE appropriately, and $13(25 \%)$ used STROBE inappropriately. 
Table 1 Overview of Strengthening the Reporting of Observational Studies in Epidemiology (STROBE) publications ordered by amount of citations received

\begin{tabular}{|c|c|c|c|c|}
\hline STROBE publication (journals) & No of citations & Impact factor* & Language & Date of publication \\
\hline The Lancet & 110 & 30.8 & English & October 2007 \\
\hline Ann Intern Med & 99 & 16.2 & English & October 2007 \\
\hline Ann Intern Med (E\&E)† & 97 & 16.2 & English & October 2007 \\
\hline$B M J$ & 69 & 13.7 & English & October 2007 \\
\hline Plos Med (E\&E)† & 62 & 13.1 & English & October 2007 \\
\hline J Clin Epidemiol & 51 & 3.0 & English & October 2008 \\
\hline Plos Med & 43 & 13.1 & English & October 2007 \\
\hline Epidemiology $(\mathrm{E} \& \mathrm{E}) \dagger$ & 42 & 5.5 & English & November 2007 \\
\hline Epidemiology & 21 & 5.5 & English & November 2007 \\
\hline Prev Med & 15 & 3.2 & English & October 2007 \\
\hline Bull World Health Organ & 14 & 5.3 & English & November 2007 \\
\hline Gaceta Sanitaria & 7 & 1.2 & Spanish & March 2008 \\
\hline Internist & 4 & 0.3 & German & June 2008 \\
\hline Gaceta Sanitaria (E\&E)† & 3 & 1.2 & Spanish & March 2009 \\
\hline Rev Esp Salud Publica & 3 & - & Spanish & May 2008 \\
\hline Notfall \& Rettungsmedizin & 0 & 0.6 & German & May 2008 \\
\hline
\end{tabular}

*Impact factors retrieved from 2009 Journal Citation Report Science Edition.

†Publication of the explanation and elaboration (E\&E) document that accompanied the STROBE recommendations.

Finally, out of the 26 studies which used STROBE appropriately, $13(50 \%)$ had authors affiliated with epidemiology or public-health departments, compared with six (46\%) among the 13 studies with inappropriate use of STROBE. As for the 10 systematic reviews that inappropriately used STROBE as a tool to assess study quality, none were published in the Cochrane Database of Systematic Reviews, nine were published in medical specialist journals, five had at least one of the authors affiliated with epidemiology or public health departments, and all were published in English. The 10 articles related to cardiology, endocrinology, psychology, neurology, paediatrics, ophthalmology, internal medicine, periodontology, pharmacology and infectious diseases.

\section{DISCUSSION}

STROBE has been cited over 600 times since its publication in 2007. Our citation analysis showed that most of the observational studies used STROBE as a guideline for reporting, whereas about half of the systematic reviews and meta-analyses used STROBE inappropriately, as a methodological quality-assessment tool.

The inappropriate interchangeable use of the terms 'methodological quality' and 'reporting quality' is common and may explain why some researchers used STROBE as a tool for the assessment of methodological quality or as a guideline to design and conduct observational research. Reporting quality refers to the completeness with which a study is presented and whether major items for the proper appraisal of internal and external validity of findings are clearly reported. ${ }^{17}$ Methodological quality refers to the appropriateness of the methods employed in the design and conduct of epidemiological research, which determines the reliability of findings (ie, internal validity).

Even though the authors of STROBE clearly state that its purpose is to guide reporting of observational research, we found that it is commonly used as a methodological quality-assessment tool in systematic reviews

Table 2 Characteristics of 51 classifiable articles citing Strengthening the Reporting of Observational Studies in Epidemiology (STROBE)

\begin{tabular}{lllc}
\hline Classification & Reason for citation & $\begin{array}{l}\text { Observational } \\
\text { research }\end{array}$ & $\begin{array}{c}\text { Systematic review, } \\
\text { meta-analysis }\end{array}$ \\
\hline Appropriate & Guideline for reporting of study & 26 & 0 \\
Inappropriate & Guideline for design and conduct of study & 3 & 0 \\
& Tool to assess methodological quality & 0 & 10 \\
& Tool to assess reporting quality & 0 & 5 \\
& Example to stress the importance of reporting guidelines & 0 & 1 \\
& Other & 2 & 1 \\
& Unclear & 1 & 2 \\
& Total & 32 & 19 \\
\hline
\end{tabular}

*This category includes observational studies which cited STROBE as a guideline without making it clear whether the guidance related to reporting or methodology, or articles which mentioned STROBE in a commentary. 
and sometimes as a guideline to design and conduct observational studies. Some journal editors have expressed concern that the STROBE recommendations may be inappropriately used as an assessment tool to judge study quality, or that researchers may use STROBE as a guideline to set up or conduct observational studies. ${ }^{10}$ Our results show that these concerns were justified, although we feel that some items of STROBE might be useful when designing or conducting an observational study. In particular, the explanation and elaboration STROBE document (E\&E) could be useful to inform methodological decisions, especially for researchers with little formal training in epidemiology.

Although some items of STROBE may be related to risk of bias, many of the items are exclusively related to transparent reporting. For instance, the first item of STROBE asks researchers to indicate in the title of their manuscript the design of their study. Obviously, whether authors report the design of their study in the title of their manuscript will not influence the effect estimates of their study or compromise the generalisability of their findings. To illustrate the pitfalls of using STROBE as a methodological assessment tool, we refer to the last text example we provided as an example of inappropriate use of STROBE in box 1. In this example, the authors of a systematic review transformed STROBE into a scale by assigning scores to each of its items, and a final score was used to determine the eligibility of studies for inclusion into the systematic review. By doing so, authors may have introduced bias to their findings, as different tools used to score methodological quality may result in different conclusions regarding the overall appropriateness of the methods employed in a particular study. ${ }^{18}$

The misuse of STROBE as a tool to assess methodological quality may be explained by the lack of validated and accepted tools for such assessments. As a consequence, authors who want to assess methodological quality of studies may turn to reporting guidelines. The absence of reliable tools that are based on sound empirical evidence to assess the quality of observational studies was documented by Sanderson et al in $2007 .{ }^{19}$

There is an extensive literature indicating that the misuse of findings of scientific articles is common across different fields of healthcare. ${ }^{720-29}$ In a similar analysis, Moher et al reported that the Consolidated Standards of Reporting Trials statement, a reporting guideline for randomised controlled trials, has also been misused by $4-10 \%$ of assessed articles as a guideline for quality assessment. ${ }^{7}{ }^{30}$ Taken together, these results indicate a failure of not only authors but also peer-reviewers in recognising and avoiding misuse of guidelines. The peerreview process should ideally prevent misuses that are of major concern, in particular when misuses may dictate the outcomes of research as discussed above.

We observed an association between the numbers of citations received and the impact factor of the journal in which STROBE was published. Even though the contents of many STROBE publications are identical, researchers preferred to cite a STROBE article published in a journal with a higher impact factor. This association was also observed by Perneger, who concluded that citations received by an article are not merely explained by scientific merit. ${ }^{31}$ Although reasons directing authors' choices are not clear, for some, a high impact factor may suggest the acceptance by a broader audience. The main exception to this association was the STROBE checklist published by the Journal of Clinical Epidemiology, which was also highly cited. This may have occurred because STROBE is particularly relevant for the daily work of the readers of this journal.

We found no clear pattern regarding affiliations of authors to an institution with a methodological focus. In particular, we found no difference in affiliations between authors who used STROBE appropriately and authors who used STROBE inappropriately. Similarly, no clear patterns emerged when we examined more closely the characteristics of the 10 systematic reviews that inappropriately cited STROBE.

A limitation of our findings is the fact that we included only articles which cited STROBE. This may have resulted in selection bias, since some researchers may have used STROBE for their study but not formally cited it. Such studies could not be identified by our search strategy. However, it is unclear to us in which direction this possible selection bias may have influenced our findings.

Citations to the STROBE checklist have been steeply increasing since its first publication. STROBE is commonly used according to its original purpose, that is, a reporting guideline of observational research. However, despite the clear statement of its objective by its authors, STROBE is misused by some researchers reporting observational studies and about half of authors reporting systematic reviews, because they used it either as a tool to assess methodological quality or as a guideline on how to design and conduct observational studies. Further studies are required to define the consequences of the inappropriate use of reporting recommendations in clinical and epidemiological research, in particular the use of these checklists as an instrument to assess the methodological quality of studies.

Funding BRdC is supported by a grant from the ARCO Foundation, Switzerland. STROBE is supported by the Swiss National Science Foundation and the Schweizerische Akademie der Medizinischen Wissenschaften.

Competing interests BRdC, MC, DGA, and ME are members of the group revising the STROBE statement.

Contributors All authors conceptualised the ideas in the manuscript, and read and approved the manuscript. BRdC developed the first draft and incorporated comments from authors for successive drafts. BRdC is the guarantor of this study.

Provenance and peer review Not commissioned; externally peer reviewed.

\section{REFERENCES}

1. Glasziou $P$, Meats $E$, Heneghan $C$, et al. What is missing from descriptions of treatment in trials and reviews? BMJ 2008;336:1472-4. 
2. Moher D. Reporting research results: a moral obligation for all researchers (in English, French). Can J Anaesth 2007;54:331-5.

3. Moher D, Jones A, Lepage L; CONSORT Group (Consolitdated Standards for Reporting of Trials). Use of the CONSORT statement and quality of reports of randomized trials: a comparative before-andafter evaluation. JAMA 2001;285:1992-5

4. Plint AC, Moher D, Morrison A, et al. Does the CONSORT checklist improve the quality of reports of randomised controlled trials? A systematic review. Med J Aust 2006;185:263-7.

5. Smidt N, Rutjes AW, van der Windt DA, et al. The quality of diagnostic accuracy studies since the STARD statement: has it improved? Neurology 2006;67:792-7.

6. Prady SL, Richmond SJ, Morton VM, et al. A systematic evaluation of the impact of STRICTA and CONSORT recommendations on quality of reporting for acupuncture trials. PLoS One 2008;3:e1577.

7. Moher D, Ocampo M, Altman D, et al, eds. Citing the CONSORT Statement and Explanation and Elaboration Paper: What's it all About? Vancouver, Canada: 6th International Congress on Peer Review and Biomedical Publication, 2009.

8. STROBE Statement. Strengthening the reporting of observational studies in epidemiology. http://www.strobe-statement.org/ (accessed 15 Dec 2010).

9. von Elm E, Altman DG, Egger M, et al. Strengthening the Reporting of Observational Studies in Epidemiology (STROBE) statement: guidelines for reporting observational studies. BMJ 2007;335:806-8.

10. Editors. Probing STROBE. Epidemiology 2007;18:789-90.

11. Mariette $\mathrm{X}$, Tubach $\mathrm{F}$, Bagheri $\mathrm{H}$, et al. Lymphoma in patients treated with anti-TNF: results of the 3-year prospective French RATIO registry. Ann Rheum Dis 2010;69:400-8.

12. Wakkee M, Meijer W, Neumann HA, et al. Psoriasis may not be an independent predictor for the use of cardiovascular and anti-diabetic drugs: a 5-year prevalence study. Acta Derm Venereol 2009:89:476-83.

13. Lavados PM, Sacks C, Prina L, et al. Incidence of lobar and non-lobar spontaneous intracerebral haemorrhage in a predominantly HispanicMestizo population-the PISCIS stroke project: a community-based prospective study in Iquique, Chile. Neuroepidemiology 2010;34:214-21.

14. Onofri M, Bonanni L, Manzoli L, et al. Cohort study on somatoform disorders in Parkinson disease and dementia with Lewy bodies. Neurology 2010;74:1598-606.

15. Gratwohl A, Stern M, Brand R, et al; European Group for Blood and Marrow Transplantation and the European Leukemia Net. Risk score for outcome after allogeneic hematopoietic stem cell transplantation: a retrospective analysis. Cancer 2009;115:4715-26.

16. Potts JA, Rothman AL. Clinical and laboratory features that distinguish dengue from other febrile illnesses in endemic populations. Trop Med Int Health 2008;13:1328-40.

17. Williams HC. Cars, Consort 2010, and clinical practice. Trials 2010;11:33.

18. Juni $P$, Altman DG, Egger M. Systematic reviews in health care: Assessing the quality of controlled clinical trials. BMJ 2001;323:42-6.

19. Sanderson S, Tatt ID, Higgins JP. Tools for assessing quality and susceptibility to bias in observational studies in epidemiology: a systematic review and annotated bibliography. Int $J$ Epidemiol 2007;36:666-76.

20. Eichorn P, Yankauer A. Do authors check their references? A survey of accuracy of references in three public health journals. Am J Public Health 1987;77:1011-12.

21. de Lacey G, Record C, Wade J. How accurate are quotations and references in medical journals? BMJ (Clin Res Ed) 1985;291:884-6.

22. Evans JT, Nadjari HI, Burchell SA. Quotational and reference accuracy in surgical journals. A continuing peer review problem. JAMA 1990;263:1353-4.

23. Fenton JE, Brazier H, De Souza A, et al. The accuracy of citation and quotation in otolaryngology/head and neck surgery journals. Clin Otolaryngol Allied Sci 2000;25:40-4.

24. Gosling CM, Cameron M, Gibbons PF. Referencing and quotation accuracy in four manual therapy journals. Man Ther 2004;9:36-40.

25. Lukic IK, Lukic A, Gluncic V, et al. Citation and quotation accuracy in three anatomy journals. Clin Anat 2004:17:534-9.

26. Wright M, Armstrong JS. The ombudsman: verification of citations: fawlty towers of knowledge? Interfaces 2008;38:125-39.

27. Reddy MS, Srinivas S, Sabanayagam N, et al. Accuracy of references in general surgical journals - an old problem revisited. Surgeon 2008;6:71-5.

28. Al-Benna S, Rajgarhia P, Ahmed S, et al. Accuracy of references in burns journals. Burns 2009;35:677-80.

29. Davids JR, Weigl DM, Edmonds JP, et al. Reference accuracy in peer-reviewed pediatric orthopaedic literature. J Bone Joint Surg Am 2010;92:1155-61.

30. Moher D, Hopewell S, Schulz KF, et al. CONSORT 2010 explanation and elaboration: updated guidelines for reporting parallel group randomised trials. BMJ 2010;340:c869.

31. Perneger TV. Citation analysis of identical consensus statements revealed journal-related bias. J Clin Epidemiol 2010;63:660-4.

\section{APPENDIX 1}

Search strategy for identifying STROBE articles

\begin{tabular}{|c|c|}
\hline Step no & Search strategy* \\
\hline 1 & $\begin{array}{l}\text { Title }=(\text { Strengthening the Reporting of } \\
\text { Observational Studies in Epidemiology) AND } \\
\text { Author }=(\text { von Elm) }\end{array}$ \\
\hline 2 & $\begin{array}{l}\text { Title }=(\text { Strengthening the Reporting of } \\
\text { Observational Studies in Epidemiology) AND } \\
\text { Author=(Vandenbroucke) }\end{array}$ \\
\hline 3 & \#1 OR \#2 \\
\hline 4 & Title $=($ STREGA $)$ \\
\hline 5 & \#3 NOT \#4 \\
\hline
\end{tabular}

